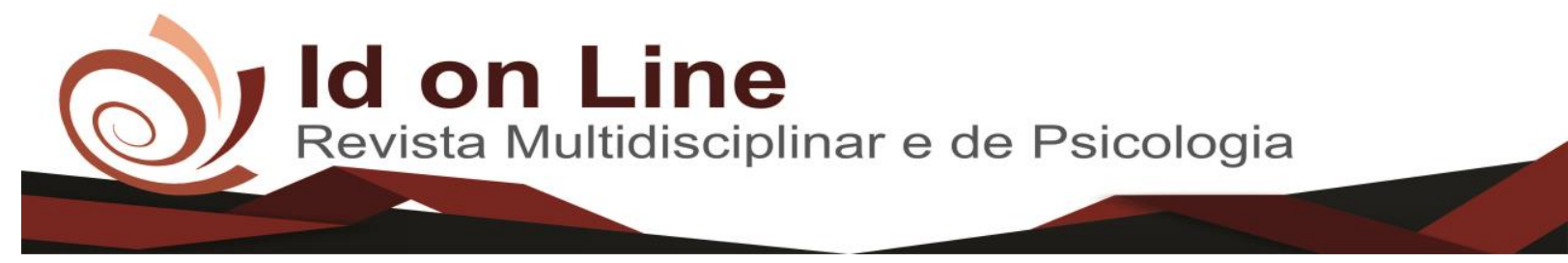

Artigo de Revisão

\title{
A Desinstitucionalização do Parto: Uma Revisão Integrativa da Literatura
}

\author{
Rozane Pereira de Sousa ${ }^{1}$; Anna Mikaelly de Sousa Tavares Assis ${ }^{2}$; Yuri Charllub Pereira Bezerra ${ }^{3 ;}$ \\ Fernanda Pereira de Brito Neves ${ }^{4}$; Gislene Farias de Oliveira ${ }^{5}$
}

\begin{abstract}
Resumo: analisar a produção científica publicada sobre a desinstitucionalização do parto. Metodo: foi realizada uma Revisão Integrativa da Literatura. Para efetivá-la, foi feito levantamento bibliográfico a partir de pesquisas indexadas na base de dados da Biblioteca Virtual em Saúde (BVS), sendo o Descritor Controlado (DeCS) em Ciências da Saúde utilizado "Parto domiciliar", identificando-se fontes de informação de inúmeras bases de literaturas científicas e técnicas. Também, foi feito agrupamento dos DeSC: Enfermagem obstétrica, parto domiciliar e tocologia. Foram identificados 116 artigos, contudo, apenas 15 deles contemplaram aos critérios de inclusão delineados. Resultados: $100 \%$ da amostra foi publicada em periódicos nacionais, sendo na sua totalidade na área da saúde. Quanto ao período de publicação evidencia-se que a maioria concentrou-se no ano de 2013 correspondendo a 40\% da amostra, seguidos por aqueles publicados em 2016 com 27\%. Os publicados nos anos de 2014 e 2015 obtiveram 20\% e 13\%, respectivamente cada. Referindo-se ao tipo de delineamento metodológico, evidenciou-se na amostra: nove estudos qualitativos $(60 \%)$; uma pesquisa bibliográfica $(6,6 \%)$; um relato de experiência $(6,6 \%)$; um estudo teórico-reflexivo $(6,6 \%)$; um estudo quantiqualitativo $(6,6 \%)$; um estudo etnográfico $(6,6 \%)$ e um estudo quantitativo $(6,6 \%)$. Em sequência, quatro artigos representando $27 \%$ da amostra, seguiram enfocando os profissionais, identificando o conhecimento e práticas do parto domiciliar; as estratégias e planejamentos utilizados para implementação e promoção do método. No mais, pela literatura apresentada, é verídico afirmar que a desinstitucionalização do parto não deve ser tratada como prática secundária, mas como um direito e uma real possibilidade de escolha da mulher e sua família por uma experiência segura e singular. Conclusão: alerta-se para a urgente necessidade da disseminação de informações acerca do parto domiciliar planejado, pois este constitui além de uma prática baseada em evidências científicas, uma possibilidade de fortalecimento da autonomia feminina e de seu protagonismo no processo de parir, baseado no direito do usuário.
\end{abstract}

Palavras-Chave: enfermagem obstétrica; parto domiciliar; tocologia.

\section{Deinstitutionalization of Childbirth: An Integrative Literature Review}

\begin{abstract}
Method: An Integrative Literature Review was carried out. To make it effective, a bibliographic survey was carried out based on surveys indexed in the Virtual Health Library (VHL) database, and the Controlled Descriptor (DeCS) in Health Sciences was used as "home birth", identifying sources of information of countless bases of scientific and technical literatures. Also, the DeSC grouping was done: Obstetric nursing, home delivery and tocology. 116 articles were identified, however, only 15 of them considered the inclusion criteria outlined. Results: $100 \%$ of the sample was published in national journals, and in its entirety in the health area. Regarding the publication period, it is evident that the majority was concentrated in the year of 2013 corresponding to $40 \%$ of the sample, followed by those published in 2016 with $27 \%$. Those published in the years of 2014 and 2015 obtained $20 \%$
\end{abstract}

\footnotetext{
${ }^{1}$ Enfermeira. Pós-Graduanda em Enfermagem Obstétrica. Faculdade Santa Maria. E-mail:rps-cefet@ hotmail.com.

${ }^{2}$ Enfermeira. Pós-Graduanda em Enfermagem Obstétrica. Faculdade Santa Maria. E-mail: mikaelly.tavares19@ @otmail.com.

${ }^{3}$ Enfermeiro. Docente FSM-PB. Mestre em Saúde Coletiva pela Universidade Católica de Santos. E-mail: yuri-m_pereira@hotmail.com.

${ }^{4}$ Médica pela Fundação de Ensino Superior de Pernambuco. Atual na Secretaria de Saúde do Estado do Ceará e como médica anestesiologista do Hospital e Maternidade São Vicente de Paulo. fpbritoneves@ hotmail.com;

${ }^{5}$ Doutora em Psicologia Social pela Universidade Federal da Paraíba - UFPB. Docente na Universidade Federal do cariri - UFCA. Contato: gislenefarias@gmail.com.
}

891 Id on Line Rev. Mult. Psic. V.12, N. 39. 2018 - ISSN 1981-1179 Edição eletrônica em http://idonline.emnuvens.com.br/id 
and $13 \%$, respectively each. Referring to the type of methodological design, nine qualitative studies $(60 \%)$ were evidenced in the sample; a bibliographic research (6.6\%); an experience report (6.6\%); a theoretical-reflexive study (6.6\%); a quantitative study (6.6\%); an ethnographic study (6.6\%) and a quantitative study (6.6\%). In sequence, four articles representing $27 \%$ of the sample, continued to focus professionals, identifying the knowledge and practices of home birth; the strategies and planning used to implement and promote the method. Moreover, from the literature presented, it is true to affirm that the deinstitutionalization of childbirth should not be treated as a secondary practice, but rather as a right and a real possibility of choosing the woman and her family for a safe and singular experience. Conclusion: it is pointed out that there is an urgent need to disseminate information about planned home birth, since it constitutes a practice based on scientific evidence, a possibility of strengthening female autonomy and its role in the process of giving birth based on right of user.

Key-words: obstetric nursing; home birth; tocology.

\section{Introdução}

A gravidez é um estado da mulher, ao qual ocorre com a união do espermatozoide com o óvulo, fecundado, após um ato sexual, que ao se fixar na mucosa uterina, pode durar em média 40 a 42 semanas, culminando com o parto ou nascimento (BEZERRA, 2014). O parto é um processo natural e fisiológico, constituindo um período vulnerável para a saúde da mulher, em que o ambiente e as práticas de assistência em saúde exercem grande influência (SUÁREZ-CORTÉS et al., 2015).

Ao longo do tempo, a história do parto e nascimento vem sendo transformada de maneira progressiva. Durante um longo período, o parto foi realizado em casa, sendo considerado um ritual de mulheres e não um ato médico, já que ficava a cargo das parteiras, porém, a partir do século XX, foi adquirindo outro significado e passou a ser considerado um procedimento que necessita de intervenções medicamentosas e cirúrgicas, que deve ser realizado por médicos, em ambiente hospitalar, institucionalizando assim o processo natural do nascimento (MALHEIROS et al., 2012).

Com a sua institucionalização, construiu-se socialmente uma visão de parto associada à doença, ao risco e sofrimento. Essa concepção ainda é a prevalente na atualidade, mesmo podendo ser nociva ao processo natural do nascimento, pois nesse modelo, o corpo da mulher é compreendido como máquina e a assistência prestada como linha de produção (SOUSA, SCHARDOSIM; 2016). 
Opondo-se ao modelo hospitalocêntrico, a desinstitucionalização da assistência ao parto, busca resgatar valores como o protagonismo, a singularidade, a privacidade e a autonomia de cada mulher. Ela promove partos saudáveis, eliminando-se as intervenções desnecessárias e oferecendo outras comprovadamente consideradas benéficas. O modelo de humanização do parto demonstra que segurança não é sinônimo de intervenção e tecnologia. Porém, pressupõe a mínima utilização de intervenção no processo fisiológico de nascimento (SANFELICE et al., 2014).

Neste contexto, embora ainda pouco conhecidos pela sociedade brasileira, às casas de parto e o parto domiciliar, apresentam relevância, já que existem evidências científicas suficientes para respaldar esta prática extra-hospitalar, demonstrando que esse tipo de parto está associado a baixas taxas de intervenções obstétricas e não há aumento nas taxas de mortalidade perinatal, reforçando que o parto domiciliar de baixo risco, planejado e assistido por profissionais capacitados, apresenta resultados favoráveis, alicerçados em um profundo respeito frente às decisões femininas (REIS et al., 2017).

Este estudo apresenta relevância social, pois, através do levantamento das evidencias existentes acerca da desinstitucionalização do parto, pode-se ampliar a visão acerca do mesmo para além dos aspectos biológicos da mulher e do bebê, focando no reconhecimento dos direitos de ambos. Dessa forma, considerando a produção científica nacional de publicações em periódicos, objetivou-se analisar a produção científica publicada sobre a desinstitucionalização do parto.

\section{Método}

Trata-se de uma revisão integrativa da literatura que compromete-se com o seguimento de critérios bem definidos sobre a coleta de dados, análise e apresentação dos resultados. Dessa forma, foram adotadas as seguintes etapas indicadas para a constituição da revisão integrativa da literatura: 1) seleção da pergunta de pesquisa; 2) definição dos critérios de inclusão de estudos e seleção da amostra; 3) representação dos estudos selecionados em formato de tabela; 4) análise crítica dos achados, identificando diferenças e conflitos; 5) 
interpretação dos resultados e 6) reportar, de forma clara, a evidência encontrada (MENDES, SILVEIRA, GALVÃO; 2008).

Para o levantamento bibliográfico, buscou-se artigos científicos na literatura brasileira publicados, no recorte temporal 2013 a 2017, nas seguintes bases de dados: Scientific Electronic Library Online (Scielo), Banco de dados em enfermagem (BDenf) e Literatura Latino-Americano e do Caribe de Informação em Ciências da Saúde (LILACS). Foram utilizados os descritores: parto domiciliar, tocologia e enfermagem obstétrica, de acordo com a classificação de Descritores em Ciências da Saúde (DeCS).

Foram identificados 116 estudos nas bases de dados abordadas, sendo 64 da Scielo, 42 da LILACS e 10 da BDenf. Os critérios de elegibilidade para inclusão na amostra contemplavam estudos que abordassem o tema proposto; tivessem sido publicados no recorte temporal supracitado e nos idiomas português ou inglês. A partir dos resultados da busca, foi executado o trabalho de eliminação de artigos não correlatos à temática em estudo, artigos não disponíveis de forma gratuita na íntegra e duplicidades. Após uma análise minuciosa, 15 se adequavam aos critérios de inclusão, compondo a amostra final desta revisão integrativa

Após a leitura minuciosa dos artigos, os estudos incluídos na revisão foram analisados de forma sistemática em relação aos seus títulos, autores, ano de publicação, objetivos, métodos e resultados, permitindo que o leitor análise o conhecimento pré-existente sobre o tema investigado.

\section{Resultados e Discussão}

Nesta Revisão Integrativa da Literatura, foram analisados quinze artigos que atenderam aos critérios de inclusão previamente definidos. Na sequência são discorridos os resultados alcançados com a proposta do estudo.

Na Tabela 1, são descritas as variáveis: autores, ano de publicação, título, periódicos, local e delineamento metodológico. Quanto aos artigos indexados a essa pesquisa, os resultados mostram que $100 \%$ da amostra foi publicada em periódicos nacionais (revistas brasileiras), sendo na sua totalidade na área da saúde. 
Tabela 1 - Distribuição dos artigos incluídos no estudo, segundo os Autores, Títulos, Periódicos, Ano de Publicação, Local e Delineamento.

\begin{tabular}{|c|c|c|c|}
\hline $\begin{array}{c}\text { AUTORES / ANO DE } \\
\text { PUBLICAÇÃO }\end{array}$ & TÍTULO & PERIÓDICO/LOCAL & DELINEAMENTO \\
\hline $\begin{array}{l}\text { Sanfelice CF de O, } \\
\text { Shimo AKK (2015) }\end{array}$ & $\begin{array}{l}\text { Representações sociais sobre o } \\
\text { parto domiciliar }\end{array}$ & $\begin{array}{l}\text { Esc. Anna Nery/Rio } \\
\text { de Janeiro }\end{array}$ & $\begin{array}{l}\text { Pesquisa qualitativa, } \\
\text { exploratória e } \\
\text { descritiva } \\
\end{array}$ \\
\hline de CM Castro (2015) & $\begin{array}{l}\text { Os sentidos do parto domiciliar } \\
\text { planejado para mulheres do } \\
\text { município de São Paulo, São } \\
\text { Paulo }\end{array}$ & $\begin{array}{l}\text { Cad. Saúde Coletiva/ } \\
\text { São Paulo }\end{array}$ & Pesquisa qualitativa \\
\hline $\begin{array}{l}\text { Sanfelice CF de O, } \\
\text { Shimo AKK (2014) }\end{array}$ & $\begin{array}{l}\text { Parto domiciliar: avanço ou } \\
\text { retrocesso? }\end{array}$ & $\begin{array}{l}\text { Rev. Gaúcha Enferm./ } \\
\text { Porto Alegre }\end{array}$ & Teórico-reflexivo \\
\hline Feyer et al (2013) & $\begin{array}{l}\text { Rituais de cuidado realizados } \\
\text { pelas famílias na preparação } \\
\text { para a vivência do parto } \\
\text { domiciliar planejado }\end{array}$ & $\begin{array}{l}\text { Rev. Bras. Enferm./ } \\
\text { Florianopolis }\end{array}$ & Estudo etnográfico \\
\hline Feyer et al (2013) & $\begin{array}{l}\text { Perfil de casais que optam pelo } \\
\text { parto domiciliar assistido por } \\
\text { enfermeiras obstétricas }\end{array}$ & $\begin{array}{l}\text { Esc. Anna Nery/ } \\
\text { Florianopolis }\end{array}$ & $\begin{array}{l}\text { Exploratório- } \\
\text { descritiva, de caráter } \\
\text { quantiqualitativo }\end{array}$ \\
\hline $\begin{array}{l}\text { Frank TC, Pelloso } \\
\text { SM (2013) }\end{array}$ & $\begin{array}{l}\text { A percepção dos profissionais } \\
\text { sobre a assistência ao parto } \\
\text { domiciliar planejado }\end{array}$ & $\begin{array}{l}\text { Rev. Gaúcha Enferm./ } \\
\text { Cascavel }\end{array}$ & $\begin{array}{l}\text { Descritivo com } \\
\text { abordagem } \\
\text { qualitativa }\end{array}$ \\
\hline Feyer et al (2013) & 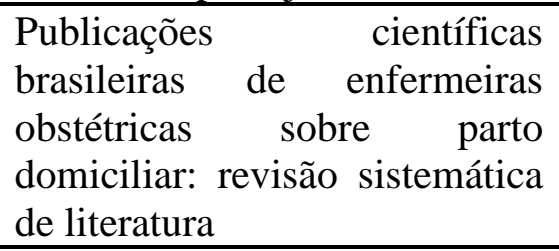 & $\begin{array}{l}\text { Texto e Contexto } \\
\text { enferm./ Florianópolis }\end{array}$ & $\begin{array}{lr}\text { Revisão } & \text { sistemática } \\
\text { do } & \text { tipo } \\
\text { metaetnografia } & \end{array}$ \\
\hline $\begin{array}{lr}\text { Koettker } & \text { JG; } \\
\text { Brüggemann } & \text { OM; } \\
\text { Dufloth RM (2013) }\end{array}$ & $\begin{array}{l}\text { Partos domiciliares planejados } \\
\text { assistidos por enfermeiras } \\
\text { obstétricas: transferências } \\
\text { maternas e neonatais }\end{array}$ & $\begin{array}{l}\text { Rev. Esc. Enf./ Santa } \\
\text { Catarina }\end{array}$ & $\begin{array}{l}\text { Estudo exploratório- } \\
\text { descritivo com } \\
\text { abordagem } \\
\text { quantitativa }\end{array}$ \\
\hline Sanfelice et al (2014) & $\begin{array}{l}\text { Do parto institucionalizado ao } \\
\text { parto domiciliar }\end{array}$ & $\begin{array}{lll}\text { Rev. } & \text { RENE/ } & \text { São } \\
\text { Paulo } & & \end{array}$ & $\begin{array}{l}\text { Estudo descritivo de } \\
\text { abordagem } \\
\text { qualitativa na } \\
\text { modalidade relato de } \\
\text { experiência }\end{array}$ \\
\hline $\begin{array}{l}\text { Souza RM, Soares } \\
\text { LS, Quitete JB } \\
(2014)\end{array}$ & $\begin{array}{l}\text { Parto natural domiciliar: um } \\
\text { poder da natureza feminina e } \\
\text { um desafio para a enfermagem } \\
\text { obstétrica }\end{array}$ & $\begin{array}{l}\text { Rev. Pesqui. cuid. } \\
\text { Fund. ./ Paraiba }\end{array}$ & $\begin{array}{l}\text { Abordagem } \\
\text { qualitativa usando o } \\
\text { método de história } \\
\text { de vida }\end{array}$ \\
\hline Barbosa et al (2013) & $\begin{array}{l}\text { Mulheres e parteiras } \\
\text { tradicionais: práticas de } \\
\text { cuidado durante o processo de } \\
\text { parto e nascimento em }\end{array}$ & $\begin{array}{l}\text { Rev. Pesqui. cuid. } \\
\text { Fund./ Paraiba }\end{array}$ & $\begin{array}{l}\text { Pesquisa qualitativa } \\
\text { que utilizou como } \\
\text { metodologia a } \\
\text { História Oral }\end{array}$ \\
\hline
\end{tabular}




\begin{tabular}{|c|c|c|c|}
\hline & domicílio & & Temática \\
\hline $\begin{array}{l}\text { Nascimento et al } \\
(2016)\end{array}$ & $\begin{array}{l}\text { O empoderamento da mulher } \\
\text { no parto domiciliar planejado }\end{array}$ & $\begin{array}{l}\text { Rev.Enferm UFPE on } \\
\text { line/ Goiás }\end{array}$ & $\begin{array}{l}\text { Estudo exploratório } \\
\text { e descritivo, com } \\
\text { abordagem } \\
\text { qualitativa. }\end{array}$ \\
\hline Moraes et al (2016) & $\begin{array}{l}\text { A dor do parto: percepção de } \\
\text { mulheres que pariram no } \\
\text { Domicílio }\end{array}$ & $\begin{array}{l}\text { Rev.Enferm UFPE on } \\
\text { line/ Goiás }\end{array}$ & $\begin{array}{l}\text { Estudo exploratório } \\
\text { e descritivo, com } \\
\text { abordagem } \\
\text { qualitativa. }\end{array}$ \\
\hline $\begin{array}{l}\text { Matão MEL et al } \\
\text { (2016) }\end{array}$ & $\begin{array}{l}\text { A visão médica do parto } \\
\text { domiciliar: factível ou utópico? }\end{array}$ & RECOM / Goiás & $\begin{array}{l}\text { Estudo descritivo, } \\
\text { abordagem } \\
\text { qualitativa }\end{array}$ \\
\hline $\begin{array}{l}\text { Mattos DV de, } \\
\text { Vandenberghe L, } \\
\text { Martins CA. }(2016)\end{array}$ & $\begin{array}{l}\text { O enfermeiro obstetra no parto } \\
\text { domiciliar planejado }\end{array}$ & $\begin{array}{l}\text { J Nurse UFPE on line/ } \\
\text { Santa } \\
\text { Catarina, Minas } \\
\text { Gerais, Rio de Janeiro, } \\
\text { São } \\
\text { Paulo, Distrito } \\
\text { Federal, Bahia, Ceará } \\
\text { e Pará }\end{array}$ & $\begin{array}{l}\text { Estudo descritivo- } \\
\text { exploratório com } \\
\text { abordagem } \\
\text { qualitativa }\end{array}$ \\
\hline
\end{tabular}

Fonte: Dados da pesquisa, 2017

Referenciando à distribuição dos artigos incluídos na pesquisa segundo o número de autores, identifica-se que prevaleceu a quantidade de quatro ou mais autores constituindo 47\%; seguido de três autores com 27\%, e três e um com 20\% e 6\% da amostra, respectivamente.

Quanto ao período de publicação evidencia-se que a maioria concentrou-se no ano de 2013 correspondendo a 40\% da amostra, seguidos por aqueles publicados em 2016 com $27 \%$. Os publicados nos anos de 2014 e 2015 obtiveram 20\% e 13\%, respectivamente cada. Observa-se que as publicações são recentes e, mesmo que no Brasil o parto domiciliar seja uma prática ainda pouco disseminada, atualmente, integra as estatísticas oficiais de nascimentos. Desde 2009, a declaração de nascimento emitida por enfermeiras obstétricas passou a ser reconhecida, alimentando o Sistema Informação sobre Nascidos Vivos (BRASIL, 2009).

Referindo-se ao tipo de delineamento metodológico, evidenciou-se na amostra: nove estudos qualitativos (60\%); uma pesquisa bibliográfica $(6,6 \%)$; um relato de experiência $(6,6 \%)$; um estudo teórico-reflexivo (6,6\%); um estudo quantiqualitativo $(6,6 \%)$; um estudo etnográfico $(6,6 \%)$ e um estudo quantitativo $(6,6 \%)$. A pesquisa de natureza qualitativa 
responde a questões muito particulares e se preocupa com um nível de realidade não possível de quantificar, pois trabalha com um universo de significados, motivos, inspirações, crenças, valores e atitudes, ou seja, com um ambiente intenso de afinidades, métodos e feitos, que não podem ser abreviados à operacionalização de variáveis (MINAYO, 2004).

Em sequencia, quatro artigos representando $27 \%$ da amostra, seguiram enfocando os profissionais, identificando o conhecimento e práticas do parto domiciliar; as estratégias e planejamentos utilizados para implementação e promoção do método. Os profissionais devem estar preparados para enfrentar as mais diversas dificuldades em relação às limitações do ambiente domiciliar, precisando estar aptos para auxiliar e orientar a parturiente e a família em todo o processo.

Estudos observacionais sugerem que o parto domiciliar em gestantes de baixo risco se associa a uma menor taxa de intervenções médicas, sem aumento da chance de efeitos adversos maternos ou neonatais (PORTO; AMORIM; SOUZA; 2010).

As contribuições são:

1. Sanfelice; Shimo (2015): evidenciou-se que as participantes mostraram-se discordantes com o modelo de atendimento institucionalizado da atualidade e buscam o parto domiciliar como uma alternativa concreta de contemplação às suas expectativas, as quais estão fortemente alicerçadas pelo princípio da autonomia.

Galvão e Menezes (2011) também observaram que as mulheres optaram pelo Parto Domiciliar pela possibilidade de experienciar sensações que incluem liberdade, integralidade, subjetividade e segurança física e emocional, que estão intimamente ligadas à confiança que possuem em suas capacidades femininas de dar à luz.

2. Castro (2015): os resultados indicam que a recusa em submeterem-se ao modelo hegemônico de assistência obstétrica vigente na maioria dos hospitais do país permitiu que as mulheres desconstruíssem a ideia do hospital como o lugar do parto. Essa recusa tem assumido um caráter de enfrentamento que exige força das mulheres e de seus companheiros, que mobilizam recursos próprios — emocionais e financeiros para defender o direito de escolher o local de parto que lhes parece mais adequado aos seus desejos e a suas necessidades.

No nosso País o enfrentamento do modelo de parto socialmente imposto e a luta pelo direito de escolha da melhor forma e local para parir ainda se configuram como um grande desafio para a mulher contemporânea, consciente de seus direitos sexuais e reprodutivos, 
sendo necessário a disseminação de informações tanto no meio cientifico como nos veículos de comunicação em geral.

3. Sanfelice; Shimo (2014): constatou-se resultados obstétricos e neonatais favoráveis ao parto domiciliar, risco semelhante quando comparado ao parto hospitalar e maiores índices de satisfação materna, fatores que legitimam a sua prática.

4. Feyer et al. (2013): os resultados revelam que a construção do ideário do parto em casa é permeada por uma rede de símbolos e significados que liga a gestação a um evento esperado da fase reprodutiva e que faz parte de um ciclo da vida que esses casais e famílias consideram como "natural", assumindo uma postura diferenciada do que postula o atual modelo de atenção ao parto e nascimento oferecido pelas instituições hospitalares.

5. Feyer et al. (2013): os resultados esclarecem que a opção pelo parto em casa está atrelada à revalorização do ambiente doméstico, e não a um resgate do passado. Os casais que optam por essa prática têm, na sua maioria, formação universitária, o que reflete na facilidade de acesso à informação e ao conhecimento biomédico, permitindo análise crítica às práticas obstétricas e para argumentar e sustentar sua decisão pelo parto em casa. Corroborando Lessa et al (2014), observam que o acesso à informação deve-se principalmente à condição educacional das mulheres, e surge como fator influenciador na tomada de consciência e na opção pelo parto domiciliar planejado.

6. Frank; Pelloso (2013): demonstram que o domicílio, enquanto local de assistência, possibilita o protagonismo da mulher e da família pela tranquilidade, calma e autonomia. O ambiente é seguro quando se segue requisitos como baixo risco gestacional, avaliação adequada, no decorrer da evolução do parto, presença de materiais adequados, rede transdisciplinar e local pré-definido para encaminhamentos. Os profissionais apontam, ainda, como fundamental, a participação familiar no processo.

7. Feyer et al. (2013): os resultados assinalam carência de publicações sobre a atuação da enfermeira obstétrica e de outros profissionais que atuam na assistência ao parto domiciliar, que possam apontar as facilidades e dificuldades encontradas neste cenário assistencial. Leão et al (2013), reforçam a necessidade de Investigações científicas para identificar e compreender as experiências de parto das mulheres, a fim de revelar 
como contribuem para o exercício da autonomia nesse processo e para evitar cesarianas indesejadas e desnecessárias.

8. Koettker; Brüggemann; Dufloth (2013): constatou-se que o parto domiciliar planejado assistido por enfermeiras obstétricas, com protocolo assistencial, apresentou bons resultados maternos e neonatais, mesmo quando a transferência para o hospital foi necessária. As evidências demonstram que as mulheres que planejaram o parto em casa tiveram uma menor incidência de parto vaginal instrumental, cesariana, analgesia peridural, transfusão de sangue, episiotomia e lacerações de $3^{\circ}$ ou $4^{\circ}$ graus em comparação às mulheres que planejaram o parto em um Centro de Parto Normal intra ou peri-hospitalar (CONITEC, 2016).

9. Sanfelice et al. (2014): evidenciou-se que atender o parto em domicílio tem oferecido maior satisfação às enfermeiras, mesmo diante de diversos obstáculos, já que é possível oferecer uma assistência à mulher e ao recém-nascido que contemple tanto o conceito de integralidade como as recomendações científicas atuais.

10.Souza; Soares; Quitete (2014) afirmam que a motivação para a escolha de um parto fora do modelo institucionalizado está relacionada à multifatores como personalidade, estilo de vida, visão de mundo e experiências vivenciadas com seus ascendentes. A enfermeira obstetra foi considerada uma profissional acolhedora e competente para acompanhar o parto domiciliar transmitindo tranquilidade e segurança às parturientes.

11. Barbosa et al. (2013): revelam o ambiente familiar proporciona conforto e tranqüilidade para as gestantes, além da autonomia em escolher a forma de ganhar seu filho, uma vez que elas assumem seus papéis como protagonistas deste processo.

12. Nascimento et al. (2016): O melhor local para o parto é aquele em que a mulher se sinta segura, podendo ser no domicílio, em um Centro de Parto Normal ou em um hospital maternidade. A residência é um ambiente seguro para o nascimento, desde que seja uma decisão da mulher e de sua família e que o processo seja acompanhado por uma equipe especializada.

13. Moraes et al. (2016): Os dados revelaram que as mulheres que tiveram parto domiciliar planejado desassociam a dor do parto de sofrimento, ou seja, mostrou que o fato de passar pela "dor do parto" fez com que elas se sentissem mais fortalecidas. De acordo com Cunha, Gomes e Santos, (2012) cada ser humano reage de forma diferente ao estímulo da dor, e, no caso das parturientes o alivio da mesma depende da forma 
como ela é abordada, podendo ser amenizada tanto através da utilização de técnicas não-farmacológicas durante o período parturitivo como também do preparo emocional prévio das mulheres para viver esse momento de modo mais tranqüilo.

14. Matão et al. (2016): os resultados evidenciaram que o despreparo médico vem se arrastando por décadas quando o assunto é parto humanizado, tudo devido à institucionalização do parto e a posse do parto como ato médico e não mais da mulher. Diante deste cenário, Müller et al. (2012) alertam para a reprodução do modelo institucionalizado do parto que começa a ser construída durante o pré-natal, realizado pelo obstetra, no qual o mesmo se esquiva em compartilhar com a mulher a decisão informada sobre o tipo de parto, construindo a noção de risco, de modo a conduzir as vivências de parto para experiências o mais controladas possível.

A conduta supracitada deve ser desconstruída, uma vez que os profissionais de saúde devem agir de forma ética e solidária, informando a mulher sobre sua saúde, evitando intervenções desnecessárias e ouvindo sua opinião sobre os procedimentos indicados, de forma clara, respeitando seu saber e o conhecimento do seu corpo.

15. Mattos; Vandenberghe; Martins (2016): os autores destacaram que o parto domiciliar planejado assistido por enfermeiro atende a política de saúde. No entanto, a efetivação plena dessa prática ainda carece de resoluções específicas que garantam acesso da parturiente ao sistema público de saúde, aos serviços privados ou conveniados ao SUS, quando necessário de encaminhamento da parturiente.

Compreende-se que o envolvimento crescente do enfermeiro no parto domiciliar planejado constitui uma das estratégias que facilitam a sua implantação e disseminação, a partir de um atendimento mais humanizado e consequentemente livre de intervenções consideradas desnecessárias, gerando dessa maneira uma maior autonomia da mulher diante do parto.

\section{Conclusões}

O objetivo delineado fora alcançado permitindo constatar que, pela literatura apresentada, é verídico que a desinstitucionalização do parto não deve ser tratada como 
prática secundária, mas como um direito e uma real possibilidade de escolha da mulher e sua família por uma experiência segura e singular.

Depreende-se que a literatura nacional relacionada a desisntitucionalização ainda está em construção, porém é necessário que as publicações recebam mais atenção dos autores, editores, analistas e veículos de publicação, para que o rigor evidencie a melhoria da qualidade das publicações. Concomitantemente espera-se que o estudo possa contribuir para a realização de futuras investigações sobre o tema, seja para auxiliar na construção de conhecimentos que se apresentem como lacunas na bibliografia, ou para aprofundar saberes já gerados por profissionais da área.

Finaliza-se alertando para a urgente necessidade da disseminação de informações acerca do parto domiciliar planejado, pois este constitui além de uma prática baseada em evidências científicas, uma possibilidade de fortalecimento da autonomia feminina e de seu protagonismo no processo de parir, baseado no direito do usuário.

\section{Referências}

BARBOSA, C.M. et al . Mulheres e parteiras tradicionais: práticas de cuidado durante o processo de parto e nascimento em domicílio. R. pesq.: cuid. fundam. Online; v.5, n.1, p.3206-3220. 2013.

BEZERRA, Y.C.P. et al. Método mãe canguru: uma revisão integrativa da literatura. Fiep bulletin, V. 84, Special Edition, Article II. 2014.

BRASIL. Rede Interagencial de Informações para a Saúde (2009) - Indicadores e dados básicos (IDB-2008). Disponível em: http://www2.datasus.gov.br/DATASUS /index.php?area=02 Acesso em 10/10/2017.

CASTRO, de C.M. Os sentidos do parto domiciliar planejado para mulheres do município de São Paulo, São Paulo. Cad. Saúde Colet.Rio de Janeiro, v. 23 n.1 p.69-75. 2015.

COMITE ESTADUAL DE ESTUDOS DE MORTALIDADE MATERNA DE PERNAMBUCO Humanização do parto. Nasce o respeito : informações práticas sobres seus direitos.Recife : 2015.

CONITEC. Ministério da Saúde. Diretriz Nacional de Assistência ao Parto Normal. 2016. Disponível em: < http://conitec.gov.br/images/Consultas/2016/Relatorio_DiretrizPartoNormal_CP.pdf> Acesso em 10/10/2017. 
CUNHA, K. J. B.; GOMES, L. S. V. O vivido de mulheres no parto humanizado Revista Interdisciplinar NOVAFAPI, Teresina. v.5, n.2, p.32-38, Abr-Mai-Jun. 2012.

FEYER, I.S.S. et al. Publicações científicas brasileiras de enfermeiras obstétricas sobre parto domiciliar: revisão sistemática de literatura. Texto contexto - enferm.v.22.n 1. Florianópolis.Jan./Mar.2013

FEYER, I.S.S et al. Perfil de casais que optam pelo parto domiciliar assistido por enfermeiras obstétricas. Esc. Anna Nery .vol.17.n.2. Rio de Janeiro.Apr./June 2013

FEYER, I.S.S. et al. Rituais de cuidado realizados pelas famílias na preparação para a vivência do parto domiciliar planejado. Rev Bras Enferm. 2013

FRANK, T.C.; PELLOSO S.M. A percepção dos profissionais sobre a assistência ao parto domiciliar planejado. Rev Gaúcha Enferm. 2013

GALVÃO, M. L. S.; MENEZES, P. F. A. Parto domiciliar: uma alternativa ao modelo institucional nos centros urbanos. In: Congresso brasileiro de Enfermagem Obstétrica e neonatal, 7 e Congresso Internacional de Enfermagem Obstétrica e Neonatal, 1. 2011, Belo Horizonte. Anais.Belo Horizonte: ABENFO-MG, 2011.

KOETTKER, J.G; BRÜGGEMANN, O.M; DUFLOTH, R.M. Partos domiciliares planejados assistidos por enfermeiras obstétricas: transferências maternas e neonatais Rev. esc. enferm. USP.v.47.n1.São Paulo.Feb.2013

LEAO, M.R.C et al. Reflexões sobre o excesso de cesarianas no Brasil e a autonomia das mulheres. Ciênc. saúde coletiva v.18 n.8 Rio de Janeiro Aug. 2013

LESSA, H.F et al. Informação para a opção pelo parto domiciliar planejado: um direito de escolha das mulheres Texto contexto - enferm. V. 23 n.3 Florianópolis July/Sept. 2014

MAIA, M.B. Humanização do parto: política pública, comportamento organizacional e ethos profissional. Rio de Janeiro: Fiocruz; 2010.

MALHEIROS, P.A et al. Parto e nascimento: saberes e práticas humanizadas. Texto contexto - enferm.v.21 n.2.Florianópolis. Apr./June.2012

MATÃO, M.; MIRANDA, D.B.; COSTA, B.P. et al. A visão médica do parto domiciliar: factível ou utópico? R. Enferm. Cent. O. Min. 2016.

MATTOS, D.V.; VANDENBERGHE, L.; MARTINS, C.A. O enfermeiro obstetra no parto domiciliar planejado Rev enferm UFPE on line., Recife. 2016

MENDES, K.D.S.; SILVEIRA, R.C.C.P.; GALVÃO, C.M. Revisão integrativa: método de pesquisa para a incorporação de evidências na saúde e na enfermagem. Texto Contexto Enferm. v.17 n.4 p.758-64. 2008 
MORAES, P. Á. et al. A dor do parto: percepção de mulheres que pariram no domicílio. Rev. enferm. UFPE on line. 2016.

MULLER, E. et al. O relato de mulheres sobre partos e intervenções: Reflexões sobre saúde, direitos humanos e cidadania. João Pessoa, 2017. Disponível em: http://www.ufpb.br/evento/lti/ocs/index.php/17redor/17redor/paper/view/322. Acesso em $10 / 10 / 2017$

NASCIMENTO, J. de P. et al. O empoderamento da mulher no parto domiciliar planejado. Rev. enferm. UFPE on line; 2016.

PORTO, A.M.F.; AMORIM, M.M.R.; SOUZA, A.S.R. Assistência ao primeiro período do trabalho de parto baseada em evidências FEMINA. V. 38 n.10 .2010 .

REIS et al. Autonomia feminina no processo de parto e nascimento: revisão integrativa da literatura. Rev Gaúcha Enferm. mar; v.38 n.1 p.64677. 2017

SANFELICE, C.F.O.; SHIMO, A.K.K. Parto domiciliar: avanço ou retrocesso? Rev. Gaúcha Enferm.v.35 n.1.Porto Alegre. 2014.

n.4 p.606-613, 2015.

Representações sociais sobre o parto domiciliar. Esc Anna Nery; v.19

SANFELICE, C.F.O et al .Do parto institucionalizado ao parto domiciliar. Rev Rene. 2014 Disponível em: http://www.revistarene.ufc.br/revista/index.php/revista/article/viewFile/1561 /pdf . Acesso em 10/10/2017.

SOUZA, R.M.; SOARES, L.S.; QUITETE, J.B. Parto natural domiciliar: um poder da natureza feminina e um desafio para a enfermagem obstétrica. J. res.: fundam. care. online. jan./mar. V.6 n.1 p.118-131, 2014.

SUÁREZ-CORTÉS, M. et al. Uso e influência dos Planos de Parto e Nascimento no processo de parto humanizado. Rev. Latino-Am. Enfermagem, v.23 n.3 p.520-6. 2015.

\section{Como citar este artigo (Formato ABNT):}

SOUZA, Rozane P de; ASSIS, Anna M. de S.T.; BEZERRA, Yuri C. P.; NEVES, Fernanda P. de B.; OLIVEIRA, Gislene F. de. A Desinstitucionalização do Parto: Uma Revisão Integrativa da Literatura. Id on Line Revista Multidisciplinar e de Psicologia, 2018, vol.12, n.39, p.891-903. ISSN: 1981-1179.

Recebido: 31.01 .2018

Aceito: 01.02 .2018 